\title{
Diagnostic utility of FGF-23 in mineral bone disorder during chronic kidney disease
}

\author{
Luisa Albanese ${ }^{1}$, Gemma Caliendo ${ }^{1}$, Giovanna $D^{\prime}$ Elia $^{1}$, Luana Passariello ${ }^{1}$, Anna Maria Molinari ${ }^{1,2}$, Claudio Napoli ${ }^{3,4}$, \\ Maria Teresa Vietri ${ }^{1,2}$ \\ ${ }^{1}$ Unity of Clinical and Molecular Pathology, AOU, University of Campania "Luigi Vanvitelli", Naples - Italy \\ ${ }^{2}$ Department of Precision Medicine, University of Campania "Luigi Vanvitelli", Naples - Italy \\ ${ }^{3}$ Department of Advanced Medical and Surgical Sciences (DAMSS), University of Campania "Luigi Vanvitelli", Naples - Italy \\ ${ }^{4}$ Clinical Department of Internal Medicine and Specialistic Units, AOU, University of Campania "Luigi Vanvitelli", Naples - Italy
}

\begin{abstract}
Our data confirm that intact fibroblast growth factor 23 (iFGF-23) concentration is increased in patients with chronic kidney disease (CKD) and that it increases with disease progression (stages I-V). Therefore, iFGF-23 could be considered an early biomarker in the course of chronic kidney disease-mineral bone disorder (CKD-MBD), which has several aspects that make it potentially useful in clinical practice. The availability of an automated method for iFGF-23 assay may represent an added value in the management of the patient with CKD-MBD already from the early stages of the disease, before the increase of the routinely used laboratory parameters, 1-84 parathyroid hormone (PTH) and 25-OH-vitamin D (25-OH-vitD), which occur in more advanced stages of the disease.
\end{abstract}

Keywords: Bone density, CKD, FGF-23

\section{Introduction}

The term "chronic kidney disease-mineral bone disorder" (CKD-MBD) is defined as a systemic disorder of bone and mineral metabolism due to CKD, which occurs in the presence of one or a combination of the following conditions: alterations in laboratory parameters (calcemia, phosphoremia, parathormone, vitamin D); abnormalities in turnover, mineralization, volume, linear growth, or bone strength; vascular or soft tissue calcifications secondary to CKD (1). The three alterations present in CKD-MBD have different prevalence in patients; that is, metabolic alterations are the first to appear followed by bone alterations and vascular calcifications.

Recently, molecules that are produced by the bone and kidney have been identified. These paths play a pivotal role in the mechanisms of bone and cardiovascular alterations during CKD-MBD. Fibroblast growth factor 23 (FGF-23)/Klotho axis, in addition to the parathyroid hormone (PTH)/vitamin D

Received: August 4, 2021

Accepted: December 4, 2021

Published online: January 8, 2022

Corresponding author:

Maria Teresa Vietri

Department of Precision Medicine

University of Campania "Luigi Vanvitelli"

Via L. De Crecchio, 7

80138 Naples - Italy

mariateresa.vietri@unicampania.it axis, has a key role in the pathophysiology of CKD-MBD, as it is involved in calcium, phosphorus, and calcitriol homeostasis, as well as in mechanisms of cellular aging (2).

FGF-23 is a glycoprotein of 252 amino acids ( $32 \mathrm{kDa}$ ), encoded by the homonymous gene located on chromosome 12. FGF-23 exerts its biological activity through interaction with one of four specific receptors (FGFR 1-4) by a paracrine mechanism (3). FGF-23 is metabolized to its inactive $\mathrm{C}$-terminal and $\mathrm{N}$-terminal fragments and intact FGF-23 (iFGF23) represents its biologically active form (4). At the cellular level, FGF-23 acts by binding to an FGF-23-FGFR complex with Klotho. Although the receptors are ubiquitously expressed, Klotho expression is restricted primarily at the level of renal tubules, parathyroid glands, and choroid plexus, determining the tissue specificity of FGF-23 $(2,5)$.

FGF-23 acts at the level of renal tubules by reducing phosphorus reabsorption and inhibiting the expression of the sodium/phosphate cotransporter (Npt2a and Npt2c) on the cell membrane in the proximal tubule. It also reduces the conversion of $25-\mathrm{OH}$-vitamin $\mathrm{D}(25-\mathrm{OH}$-vitD) to its active form $1,25(\mathrm{OH})$ by inhibiting the expression of $1 \alpha$-hydroxylase and increases its degradation by increasing the activity of 24-hydroxylase.

In CKD there is a progressive increase in FGF-23 levels associated with reduced renal function. Several factors may contribute to this phenomenon, such as hyperphosphatemia, hypercalcemia, secondary hyperparathyroidism, and Klotho deficiency (6). Serum levels of FGF-23 increase in the early stages of CKD as a compensatory mechanism to prevent the onset of hyperphosphatemia and secondary 
hyperparathyroidism (7). Even transient increase in phosphoremia, in the early stages of CKD, stimulates FGF-23 production, which then tends to normalize phosphoremia but at the same time causes a reduction in $1,25(\mathrm{OH})$ vitD resulting both in increased parathormone synthesis and secretion $(8,9)$.

Extrarenal manifestations of FGF-23 in CKD can be Klothodependent and/or Klotho-independent, receptor action of FGF-23 that does not require the presence of the membrane coreceptor. Klotho-independent effects of FGF-23 in CKD are expressed at the cardiac level, with hypertrophy of left ventricular myocytes, and at the hepatic level, with increased synthesis of proinflammatory cytokines (10).

During renal failure, effects of both Klotho-dependent and -independent FGF-23 are instead expressed at the level of the central nervous system, the immune system, and the vascular system.

Enzyme immunoassays, used until now to assay FGF-23, recognized both the $\mathrm{C}$-terminal portion and the intact form of FGF-23. The current availability of a new automated chemiluminescence immunoassay (CLIA) for the determination of the intact molecule only allows overcoming these methodological limitations, favoring a routine use of the FGF-23 assay in clinical practice, in association with parameters already used routinely such as parathormone and vitamin $D$ $(11,12)$

The aim of the present descriptive study was to assess the circulating levels of iFGF-23 in patients with CKD at various stages in specialist follow-up with the Liaison iFGF-23 assay.

\section{Methods}

Sixty-three patients (35 males and 28 females, aged 20-80 years) diagnosed with CKD-MBD were selected; all underwent renal transplantation. The patients were hospitalized at the Teaching Hospital of the University of Campania "Luigi Vanvitelli" of Naples (Italy) and signed an informed consent at the admission time.

Patients aged $<18$ years, with inflammatory bowel disease, acute infectious or inflammatory disease, or advanced neoplasia were excluded from the study.

Circulating levels of iFGF-23, 1-84 PTH, 25-OH-vitD, creatinine, and albumin were assayed for each patient.

Blood samples were collected at 8 am from fasting patients, centrifuged, and stored at $-20^{\circ} \mathrm{C}$ until assayed.

1-84 PTH, 25-OH-vitD, and iFGF-23 were assayed using chemoluminescence (CLIA) methods on the LIAISON XL platform (DiaSorin spa, Italy).

Albumin and creatinine were assayed by the colorimetric method (Architect Abbott Diagnostics).

Reference values for 1-84 PTH ranged from 4.6 to $58.1 \mathrm{pg} /$ $\mathrm{mL}$, whereas for $25-\mathrm{OH}$-vitD it was $<10 \mathrm{ng} / \mathrm{mL}$ (deficiency), $10-30 \mathrm{ng} / \mathrm{mL}$ (insufficiency), $30-100 \mathrm{ng} / \mathrm{mL}$ (sufficiency).

The reference range for iFGF-23 was $25.1-95.5 \mathrm{pg} / \mathrm{mL}$; for creatinine it was $0.72-1.25 \mathrm{mg} / \mathrm{dL}$ for males and 0.57 $1.11 \mathrm{mg} / \mathrm{dL}$ for females; and for albumin it was $3.5-5.2 \mathrm{~g} / \mathrm{dL}$.

Glomerular filtrate was estimated using clearance of creatinine according to the eGFR-EPI equation for the classification of patients at a given stage of CKD. Thus, of the 63 patients with eGFR between 16 and $104 \mathrm{~mL} / \mathrm{min} / 1.73 \mathrm{~m}^{2}$,
10 had stage I, 19 stage II, 22 stage III, 7 stage IV, and 5 stage V CKD.

\section{Statistical analysis}

Data are reported as means \pm standard deviation (SD), unless otherwise stated. Comparison between the groups was performed using $\chi^{2}$ test. Significance was assumed for $\mathrm{p}$-values less than 0.05 .

\section{Results}

About $39.7 \%(25 / 63)$ of patients with CKD had iFGF-23 levels above normal (>95.5 pg/mL), 14.3\% (9/63) of patients had 1-84 PTH values increased beyond the normal range $(>58.1 \mathrm{pg} / \mathrm{mL})$, and $7.9 \%(5 / 63)$ had insufficient $25-\mathrm{OH}$-vitD values $(<10 \mathrm{ng} / \mathrm{mL})$.

Table I shows the number of patients distributed in the different stages of CKD with iFGF-23 and 1-84 PTH values above the cutoff and with 25-OH-vitD deficiency.

TABLE I - Percentage of patients with increased values of iFGF-23 and 1-84 PTH and 25-OH-vitD deficiency divided into the five stages of CKD

\begin{tabular}{|c|c|c|c|}
\hline $\begin{array}{l}\text { Patients with } \\
\text { CKD } \\
\text { (total 63) }\end{array}$ & $\begin{array}{c}\text { iFGF-23 } \\
\text { (>95.5 pg/mL) }\end{array}$ & $\begin{array}{c}1-84 \text { PTH } \\
\text { (>58.1 pg/mL) }\end{array}$ & $\begin{array}{l}\text { 25-OH-vitD } \\
\text { (<10 ng/mL) }\end{array}$ \\
\hline Stage I $(n=10)$ & $2 / 10(20 \%)$ & - & - \\
\hline Stage II $(n=19)$ & $5 / 19(26.3 \%)$ & $2 / 19$ (10.5\%) & - \\
\hline Stage III $(n=22)$ & $9 / 22$ (40.9\%) & $4 / 22(18.2 \%)$ & $2 / 22$ (9\%) \\
\hline Stage IV $(n=7)$ & $6 / 7(85.7 \%)$ & $1 / 7(14.3 \%)$ & $2 / 7(28.6 \%)$ \\
\hline Stage $V(n=5)$ & $3 / 5(60 \%)$ & $2 / 5(40 \%)$ & $1 / 5$ (20\%) \\
\hline
\end{tabular}

$\mathrm{CKD}=$ chronic kidney disease; $\mathrm{FFGF}-23=$ intact fibroblast growth factor 23 ; $\mathrm{PTH}=$ parathyroid hormone; $25-\mathrm{OH}$-vitD $=25-\mathrm{OH}$-vitamin $\mathrm{D}$.

Specifically, $20 \%$ of stage I, $26.3 \%$ of stage II, $40.9 \%$ of stage III, $85.7 \%$ of stage IV, and $60 \%$ of stage V CKD patients had iFGF-23 levels above the cutoff. No statistically significant differences are found among the CKD stages $(p<0.05)$.

Values of 1-84 PTH above the normal range were observed in $10.5 \%$ of stage II, $18.2 \%$ of stage III, and $14.3 \%$ and $40 \%$ of stage IV and stage V CKD patients, respectively. For stage I CKD patients, increased values of 1-84 PTH were not found.

In addition, insufficient $25-\mathrm{OH}$-vitD values were found in $9 \%, 28.6 \%$, and $20 \%$ of patients at CKD stages III, IV, and V, respectively.

Table II shows the percentages of patients with increased serum values of iFGF-23,1-84 PTH, and 25-OH-vitD deficiency divided according to the number of years since transplantation ( $<10$ years, $11-20$ years, and $>20$ years).

Thirty-two of the 63 patients had received transplantation for $<10$ years, 20 for $11-20$ years, and 11 for $>21$ years.

Values of iFGF-23 beyond the normal range were found in $43.7 \%$ of organ transplant patients received less than 10 years, 35\% received between 11 and 20 years, and 36\% received $>20$ years. 
TABLE II - Percentage of patients with elevated iFGF-23 and 1-84 PTH levels and 25-OH-vitD deficiency according to years since transplantation

\begin{tabular}{lccc}
\hline $\begin{array}{l}\text { Renal } \\
\text { Transplantation }\end{array}$ & $\begin{array}{c}\text { iFGF-23 } \\
(>\mathbf{9 5 . 5} \mathbf{~ p g} / \mathbf{m L})\end{array}$ & $\begin{array}{c}\mathbf{1 - 8 4} \mathbf{P T H} \\
(\mathbf{> 5 8 . 1} \mathbf{~ p g} / \mathbf{m L})\end{array}$ & $\begin{array}{c}\mathbf{2 5 - O H - v i t D} \\
(<\mathbf{1 0} \mathbf{~ n g} / \mathbf{m L})\end{array}$ \\
\hline$<10$ years $(\mathrm{n}=32)$ & $14 / 32(43.7 \%)$ & $6 / 32(18.7 \%)$ & $3 / 32(9.4 \%)$ \\
$11-20$ years $(\mathrm{n}=20)$ & $7 / 20(35 \%)$ & $2 / 20(10 \%)$ & $2 / 20(10 \%)$ \\
$>20$ years $(\mathrm{n}=11)$ & $4 / 11(36 \%)$ & $1 / 11(9.1 \%)$ & - \\
\hline
\end{tabular}

iFGF-23 = intact fibroblast growth factor 23; PTH = parathyroid hormone; 25-OH-vitD $=25-\mathrm{OH}$-vitamin D.

Increased values of 1-84 PTH have been detected in $18.7 \%$ of transplant patients received less than 10 years, $10 \%$ of transplant patients received $11-20$ years, and $9.1 \%$ of transplant patients received more than 20 years.

A $25-\mathrm{OH}$-vitD deficiency existed in $9.4 \%$ of patients who had received transplantation for less than 10 years and $10 \%$ of patients who had received transplantation for more than 11 years. No deficient 25-OH-vitD values were found for patients who had been transplanted for more than 20 years.

\section{Discussion}

Increased serum FGF-23 levels from the earliest stages of CKD represent the first indicator of alterations in mineral metabolism. Increased levels of FGF-23, 1-84 PTH, and phosphorus with a concomitant reduction in serum levels of active vitamin $\mathrm{D}$ are closely related to reduced glomerular filtration rate (GFR).

The increase in FGF-23 concentration may be caused by the abnormal stimulation of its secretion exerted by substances released from the damaged renal parenchyma or by the effects exerted by the uremic state on bone mineralization processes. Other shreds of evidence, instead, suggest the crucial role that phosphorus plays in the uncontrolled stimulation of FGF-23 secretion (3). Limitation of our study is the distribution of samples by disease stage. Out of 63 enrolled patients, 41 had CKD stages II-III.

Despite this limitation, our results show increased FGF-23 levels in a high percentage of patients with CKD, which is in line with what has been recently reported (12). Moreover, our data show that the percentage of patients with iFGF-23 values above reference limits increases with disease progression (stages I-V).

In the early stages of CKD, increasing iFGF-23 could represent a compensatory mechanism to prevent the establishment of hyperphosphatemia and secondary hyperparathyroidism. Starting from stage IV, with a further reduction of residual renal function, Klotho levels are also reduced, resulting in peripheral resistance due to the FGF-23 action and further increase in the iFGF-23 levels.

Renal transplantation represents the therapy of choice in CKD, allowing, compared to dialysis, a better quality of life and increased survival (13-15).

Indeed, it is reported that after renal transplantation the concentration of FGF-23 decreases rapidly. Here, we noticed a correlation between plasma iFGF-23 concentration and years since transplantation. Indeed, iFGF-23 values above the normal limit were found in $43.7 \%$ of patients transplanted less than 10 years, in 35\% of patients transplanted $10-20$ years, and in $36 \%$ of patients who had received transplantation for more than 20 years.

In addition, increased levels of 1-84 PTH have been observed in $18.7 \%$ of patients after renal transplantation less than 10 years while $10 \%$ of patients with transplantation for $10-20$ years, and $9 \%$ of patients with transplantation for more than 20 years. These data indicate, as has been pointed out, that after renal transplantation, persistent hyperparathyroidism is associated with elevated iFGF-23 levels, which may occur with inhibition of the synthesis of the active form of vitamin $D$, resulting in the persistence of elevated levels of 1-84 PTH.

The persistence of high levels of iFGF-23 after renal transplantation can be interpreted as a mechanism of compensation and adaptation to the new metabolic balance created in the early posttransplant phases. Factors that may favor the persistence of high levels of FGF-23 after transplantation may include immunosuppressive therapy: corticosteroids, calcineurin inhibitors, and mammalian target of rapamycin (mTOR) inhibitors, which stimulate the production of iFGF-23 (16).

\section{Conclusions}

The availability of an automated method for iFGF-23 assay may represent an added value in the management of the patient with CKD-MBD already from the early stages of the disease, before the increase of the routinely used laboratory parameters, 1-84 PTH and 25-OH-vitD, which occur in more advanced stages of the disease.

\section{Disclosures}

Conflict of interest: The authors declare no conflict of interest. Financial support: This research received no specific grant from any funding agency in the public, commercial, or not-for-profit sectors. Authors contribution: all authors contributed equally to this manuscript.

\section{References}

1. Moe S, Drüeke T, Cunningham J, et al; Kidney Disease: Improving Global Outcomes (KDIGO). Definition, evaluation, and classification of renal osteodystrophy: a position statement from Kidney Disease: Improving Global Outcomes (KDIGO). Kidney Int. 2006;69(11):1945-1953. CrossRef PubMed

2. Hu MC, Shiizaki K, Kuro-o M, Moe OW. Fibroblast growth factor 23 and Klotho: physiology and pathophysiology of an endocrine network of mineral metabolism. Annu Rev Physiol. 2013;75(1):503-533. CrossRef PubMed

3. Ho BB, Bergwitz C. FGF23 signalling and physiology. J Mol Endocrinol. 2021;66(2):R23-R32. CrossRef PubMed

4. Shimada T, Urakawa I, Isakova T, et al. Circulating fibroblast growth factor 23 in patients with end-stage renal disease treated by peritoneal dialysis is intact and biologically active. $J$ Clin Endocrinol Metab. 2010;95(2):578-585. CrossRef PubMed

5. Angelin B, Larsson TE, Rudling M. Circulating fibroblast growth factors as metabolic regulators - a critical appraisal. Cell Metab. 2012;16(6):693-705. CrossRef PubMed 
6. Wolf M. Update on fibroblast growth factor 23 in chronic kidney disease. Kidney Int. 2012;82(7):737-747. CrossRef PubMed

7. Bergwitz $\mathrm{C}$, Jüppner $\mathrm{H}$. Regulation of phosphate homeostasis by PTH, vitamin D, and FGF23. Annu Rev Med. 2010;61(1): 91-104. CrossRef PubMed

8. Isakova T, Wahl P, Vargas GS, et al. Fibroblast growth factor 23 is elevated before parathyroid hormone and phosphate in chronic kidney disease. Kidney Int. 2011;79(12):1370-1378. CrossRef PubMed

9. Isakova T, Xie H, Yang W, et al; Chronic Renal Insufficiency Cohort (CRIC) Study Group. Fibroblast growth factor 23 and risks of mortality and end-stage renal disease in patients with chronic kidney disease. JAMA. 2011;305(23):2432-2439. CrossRef PubMed

10. Takashi Y, Fukumoto S. FGF23 beyond phosphotropic hormone. Trends Endocrinol Metab. 2018;29(11):755-767. CrossRef PubMed

11. Shimizu Y, Fukumoto S, Fujita T. Evaluation of a new automated chemiluminescence immunoassay for FGF23. J Bone Miner Metab. 2012;30(2):217-221. CrossRef PubMed
12. Souberbielle JC, Prié D, Piketty ML, et al. Evaluation of a new fully automated assay for plasma intact FGF23. Calcif Tissue Int. 2017;101(5):510-518. CrossRef PubMed

13. Napoli C, Casamassimi A, Crudele V, Infante T, Abbondanza C. Kidney and heart interactions during cardiorenal syndrome: a molecular and clinical pathogenic framework. Future Cardiol. 2011;7(4):485-497. CrossRef PubMed

14. Vasco M, Benincasa G, Fiorito C, et al. Clinical epigenetics and acute/chronic rejection in solid organ transplantation: an update. Transplant Rev (Orlando). 2021;35(2):100609. CrossRef PubMed

15. Bellastella G, Maiorino MI, De Bellis A et al. Serum but not salivary cortisol levels are influenced by daily glycemic oscillations in type 2 diabetes. Endocrine. 2016;53(1):220-6. CrossRef. PubMed

16. Economidou D, Dovas S, Papagianni A, Pateinakis P, Memmos D. FGF-23 levels before and after renal transplantation. J Transplant. 2009;2009:379082. CrossRef PubMed 